INPLASY

PROTOCOL

To cite: Lima et al. Is changes in shoulder muscle strength a risk factor for shoulder pain in volleyball athletes? A systematic review. Inplasy protocol 202160040. doi: 10.37766/inplasy2021.6.0040

Received: 13 June 2021

Published: 13 June 2021

Corresponding author: Alane Lima

alane.almeida@hotmail.com

Author Affiliation:

Federal University of Ceara.

Support: None.

Review Stage at time of this submission: Piloting of the study selection process.

Conflicts of interest:

None declared.

\section{Is changes in shoulder muscle strength a risk factor for shoulder pain in volleyball athletes? A systematic review}

Lima, $A A^{1}$; de Jesus, $E R^{2}$; Simim, MAM³; Oliveira, $R^{4}$; Medeiros, AIA 5 .

Review question / Objective: Is changes in shoulder muscle strength a risk factor for shoulder pain in volleyball athletes? Condition being studied: Shoulder pain.

Eligibility criteria: Inclusion: i) Study published as original research in a peer-reviewed journal as a full-text article; ii) Study of cross-sectional, prospective cohort; iii) Study carried out on volleyball or beach volleyball; Exclusion: i) Study performed on retired athletes; ii) Study that mixed other sports with volleyball and not differ results for each one.

INPLASY registration number: This protocol was registered with the International Platform of Registered Systematic Review and Meta-Analysis Protocols (INPLASY) on 13 June 2021 and was last updated on 13 June 2021 (registration number INPLASY202160040).

\section{INTRODUCTION}

Review question / Objective: Is changes in shoulder muscle strength a risk factor for shoulder pain in volleyball athletes?

Condition being studied: Shoulder pain.

\section{METHODS}

Search strategy: Four electronic databases (PubMed, Web of Science, SPORTDiscus, and Scopus) was systematically searched from their respective dates of inception up to Maio de 2021 using combinations of the following terms: ("Shoulder" OR 
"Glenoumeral” OR "Upper limb" OR "upper extremity" OR "arm" OR "rotator cuff" OR "rotator muscles" OR "scapular muscle") AND ("Strength" OR "weakness" OR "muscular imbalance" OR "risk factor" OR "preseason" OR "isokinetic" OR "isometric" OR "hand held" OR "dynamometer" OR "torque") AND ("volleyball" OR "beach volleyball") AND ("shoulder pain" OR "shoulder injury" OR "Shoulder Impingement" OR "rotator cuff injury")Only English language studies will be included.

Participant or population: Volleyball athletes.

Intervention: Strength assessment of shoulder muscles.

Comparator: Contralateral limb or control group.

Study designs to be included: crosssectional and propectives cohort studies.

Eligibility criteria: Inclusion: i) Study published as original research in a peerreviewed journal as a full-text article; ii) Study of cross-sectional, prospective cohort; iii) Study carried out on volleyball or beach volleyball; Exclusion: i) Study performed on retired athletes; ii) Study that mixed other sports with volleyball and not differ results for each one.

Information sources: We will source information from the papers, if it is not presented, we will contact the authors.

Main outcome(s): Shoulder pain.

Quality assessment / Risk of bias analysis: We will use an adaptation of Joanna Brigg Intitute's critical appraisal check list for studies repporting prevalence date.

Strategy of data synthesis: Findings will be reported in a paper under the Preferred Reporting Items for Systematic Reviews and Meta-Analyses recommendations (PRISMA).
Subgroup analysis: Due to methodological heterogeneity, we will perform the analysis in subgroups considering the type of contraction. Participants will be divided into: concentric, eccentric and isometric. We will also evaluate in subgroups according to the type of study: crosssectional and prospective.

Sensitivity analysis: We will evaluate the effect size.

Language: English.

Country(ies) involved: Brazil.

Keywords: shoulder; strength; volleyball; risk factor.

Contributions of each author:

Author 1 - Alane Lima.

Email: alane.almeida@hotmail.com

Author 2 - Eric Jesus.

Email: dejesuseric1310@gmail.com

Author 3 - Mario Simim.

Email: mario.simim@ufc.br

Author 4 - Rodrigo Oliveira.

Email: rodrigoroliveira@hotmail.com

Author 5 - Alexandre Medeiros.

Email: alexandremedeiros@ufc.br 